\title{
Ruin Probability and Time of Ruin with a Proportional Reinsurance Threshold Strategy
}

\author{
Anna Castañer · M. Mercè Claramunt . \\ Maite Mármol
}

\begin{abstract}
In this paper we present a threshold proportional reinsurance strategy and we analyze the effect on some solvency measures: ruin probability and time of ruin. This dynamic reinsurance strategy assumes a retention level that is not constant and depends on the level of the surplus. In a model with inter-occurrence times generalized $\operatorname{Erlang}(n)$-distributed we obtain the integro-differential equation for the Gerber-Shiu function. Then, we present the solution for inter-occurrence times exponentially distributed and claim amount phase-type $(N)$. Some examples for exponential and phase-type(2) claim amount are presented. Finally, we show some comparisons between threshold reinsurance and proportional reinsurance.
\end{abstract}

Keywords Gerber-Shiu function · Generalized Erlang $(n) \cdot$ Reinsurance strategy · Solvency measures

Mathematics Subject Classification (2000) 62P05 · 91B30

\section{Introduction}

Studies of the effect of a reinsurance strategy on solvency measures have concentrated their attention on the ultimate ruin probability. A good number of these studies analyze the effect of reinsurance on the adjustment coefficient or Lundberg exponent as this coefficient defines an upper bound for the ruin probability with infinite time horizon. Many authors have considered the problem of determining the optimal level and/or type of reinsurance with the probability of ruin criterion (Waters 1979, 1983; Gerber 1979; Centeno 1986, 2002;

\footnotetext{
A. Castañer

Dept. Matemàtica Econòmica, Financera i Actuarial, University of Barcelona, Barcelona, Spain

E-mail: acastaner@ub.edu

M.M. Claramunt

Dept. Matemàtica Econòmica, Financera i Actuarial, University of Barcelona, Barcelona, Spain E-mail: mmclaramunt@ub.edu
}

M. Mármol ( $\)$

Dept. Matemàtica Econòmica, Financera i Actuarial, University of Barcelona, Av. Diagonal, 696, 08034, Barcelona, Spain

E-mail: mmarmol@ub.edu 
Goovaerts et al. 1989; Hesselager 1990; Bühlmann 1996; Bowers et al. 1997; Schmidli 2001, 2002; Verlaak and Beirlant 2003; Hipp and Vogt 2003; Taksar and Markussen 2003).

The reinsurance strategy considered may be static or dynamic. In the first case, it is assumed that the level and type of reinsurance remain constant throughout the period considered, which in many cases is infinite (Waters 1983; Centeno 1986, 2005; Dickson and Waters 1996). In the dynamic case, we can find papers which consider that for a fixed type of reinsurance the level of reinsurance can change continuously (Hojgaard and Taksar 1998; Schmidli 2001, 2002; Hipp and Vogt 2003; Taksar and Markussen 2003). In these papers, optimal stochastic control tools in continuous time are used. Dickson and Waters (2006) assume that the insurer can change the type and/or level of reinsurance at the start of each year, so they studied a discrete time stochastic control problem.

In this paper we consider a Sparre Andersen model, and introduce a dynamic reinsurance strategy. We assume that the insurer considers a proportional reinsurance arrangement, where the retention level is not constant and depends on the level of the surplus. We then define a threshold proportional strategy: A retention level $k_{1}$ is applied whenever the reserves are less than a specific threshold $b$, and a retention level $k_{2}$ is applied in the other case. Since, for the insurer, reinsurance is a tool for controlling the solvency of the portfolio, it seems natural that the retention level could depend on the surplus level at some point or instant. The threshold proportional reinsurance strategy that we propose in this paper is an easy and clear way to include this dependence.

The objective of this paper is to analyze the effect of this new strategy on solvency measures of the insurer using the Gerber-Shiu function, which allows us to obtain ruin probability and some characteristics of time of ruin.

The introduction of this reinsurance policy bears certain similarities to the threshold dividend strategy, introduced initially by Lin and Pavlova (2006). This dividend strategy proposes that dividends should not be paid as long as the reserves remain below a certain level $b$, and that when the reserves surpass this level a constant intensity $d$ of the premium received, $c$, should be paid out in the form of dividends. So, the introduction of the pay-out means that two premium intensities are applied: $c_{1}$, the premium for levels of reserves below $b$, and $c_{2}=(c-d)$ for levels of reserves above $b$, such that $c_{1} \geq c_{2}$.

With the introduction of the threshold reinsurance policy, the claim amount paid by the insurer is modified depending on the level of the reserves, i.e., if the claim occurs when the level of the reserves is greater than $b$, the insurer pays a percentage $k_{2}$ of the claim amount, and if it occurs when the reserves are below $b$, a percentage $k_{1}$ of the claim amount is paid. This is an important difference with Lin and Pavlova's model.

Also in our model, the fact of applying different percentages of cession of the risk to the reinsurer causes different intensities of premiums for the insurer. The model does not demand a relation of order between the percentages and so there is no need that $c_{1} \geq c_{2}$ holds.

The paper is organized as follows: In Sect. 2 we explain the assumptions and some preliminaries. In Sect. 3, we obtain the integro-differential equation for the Gerber-Shiu function in a model with a threshold reinsurance strategy and with inter-occurrence times generalized Erlang $(n)$-distributed. In the rest of the paper we concentrate on the Poisson model, so we consider that inter-occurrence times are exponentially distributed. In Sect. 4 we assume that the individual claim amount follows a phase-type $(N)$. Finally, in Sect. 5 some comparisons between threshold reinsurance and proportional reinsurance are presented. 


\section{Assumptions and preliminaries}

In the Sparre Andersen model, the surplus process, $R(t)$, at a given time $t \in[0, \infty)$ is defined as $R(t)=u+c t-S(t)$, with $u=R(0) \geq 0$ being the insurer's initial surplus, $S(t)$ the aggregate claims and $c$ the rate at which the premiums are received.

$\{S(t)\}$ is modelled as a compound process where

$$
S(t)=\sum_{i=1}^{N(t)} X_{i},
$$

$N(t)=\min \left\{k: T_{1}+\ldots+T_{k+1}>t\right\}$, the number of claims occurring until time $t$, is an ordinary renewal process, and the inter-occurrence times between claims, $\left\{T_{i}\right\}_{i=1}^{\infty}$, are modeled as a sequence of i.i.d. random variables, where $T_{1}$ denotes the time until the first claim and $T_{i}$ , for $i>1$, denotes the time between the $(i-1)$ th and $i$ th claim. Note that in a Poisson process with parameter $\lambda, T_{i}, i \geq 1$ has an exponential distribution with mean $1 / \lambda$. The claims $\left\{X_{i}, i \geq 1\right\}$ are i.i.d. random variables with density function $f(x)$, and common expectation $E[X]<\infty$.

Premiums are assumed to be payable continuously at rate $c$ per unit time where $c=$ $(1+\rho) E[X] / E\left[T_{i}\right]$ with $\rho>0$ the relative security loading (net profit condition).

In this paper, we will assume that the random variables $T_{i}, i \geq 1$ are generalized $\operatorname{Erlang}(n)$ distributed, i.e. each $T_{i}$ is a sum of $n$ independent exponential random variables with possibly different parameters $\lambda_{1}, \ldots, \lambda_{n}$.

The time of ruin is defined as $T=\inf \{t: R(t)<0\}$, with $T=\infty$ if $R(t) \geq 0$ for all $t \geq 0$. The ruin probability is defined as

$$
\psi(u)=P[T<\infty \mid R(0)=u]=E\{I(T<\infty) \mid R(0)=u\},
$$

where $I(A)=1$ if $A$ occurs and $I(A)=0$ otherwise.

Let us first consider the effect of proportional reinsurance. The ceding company (insurer) and the reinsurer agree on a cession percentage, say $(1-k), k$ being the retention level applied to each claim.

We assume that insurance and reinsurance premiums include positive loading factors, $\rho_{R}>0$ being the reinsurer's loading factor.

The premium income retained by the insurer, $c^{\prime}$, depends on $\rho_{R}$ and $k$, where

$$
c^{\prime}=\frac{E[X]}{E\left[T_{i}\right]}(1+\rho)-(1-k)\left(1+\rho_{R}\right) \frac{E[X]}{E\left[T_{i}\right]} .
$$

A new security loading for the insurer, $\rho_{N}$, can be defined. Knowing that $c^{\prime}=k(1+$ $\left.\rho_{N}\right) E[X] / E\left[T_{i}\right]$, from (1)

$$
\rho_{N}=\rho_{R}-\frac{\rho_{R}-\rho}{k}, \forall k>0 .
$$

If $\rho=\rho_{R}$, the premium paid by the policyholder $c$ is shared between insurer and reinsurer in the same proportion $k$, so $c^{\prime}=k c$ and $\rho_{N}=\rho$. It is normally assumed that $\rho_{R}>\rho$ because if $\rho>\rho_{R}$ the insurer would simply cede his entire portfolio to the reinsurers, a situation which would be nonsensical.

Let $R^{-}(T)$ be the surplus just before ruin, and $R^{+}(T)$ the surplus at ruin if ruin occurs. Gerber and Shiu $(1998,2005)$ define the function

$$
\phi(u)=E\left[e^{-\delta T} w\left(R^{-}(T),\left|R^{+}(T)\right|\right) I(T<\infty) \mid R(0)=u\right],
$$


where $\delta \geq 0$ is the discounted factor, and $w(x, y), x \geq 0, y>0$, is the penalty function, so that $\phi(u)$ is the expected discounted penalty payable at ruin. This function is known to satisfy a defective renewal equation (Gerber and Shiu 1998; Lin and Garrido 2004; Willmot 2007) but easy explicit formulae for $\phi(u)$ are only available for certain special cases for the claim size distribution (Lin and Willmot 1999, 2000; Landriault and Willmot 2008).

Let $w(x, y)=1$; we then obtain the expression for the defective Laplace transform (LT) of the time of ruin $\phi(u)=E\left[e^{-\delta T} I(T<\infty) \mid R(0)=u\right]$, and if in addition $\delta=0$, then $\phi(u)=P[T<\infty \mid R(0)=u]=\psi(u)$, i.e. the ruin probability.

In this paper, we consider a threshold proportional reinsurance strategy defined by a threshold $b \geq 0$. A retention level $k_{1}$ is applied whenever the reserves are less than $b$, and a retention level $k_{2}$ is applied otherwise. Then, the premium incomes retained are $c_{1}$ and $c_{2}$, respectively. We consider that the retention levels give new positive security loadings for the insurer, i.e. the net profit condition is always fulfilled. From (2), we can define

$$
\begin{aligned}
& \rho_{1}=\rho_{R}-\frac{\rho_{R}-\rho}{k_{1}}, \\
& \rho_{2}=\rho_{R}-\frac{\rho_{R}-\rho}{k_{2}} .
\end{aligned}
$$

Graphically,

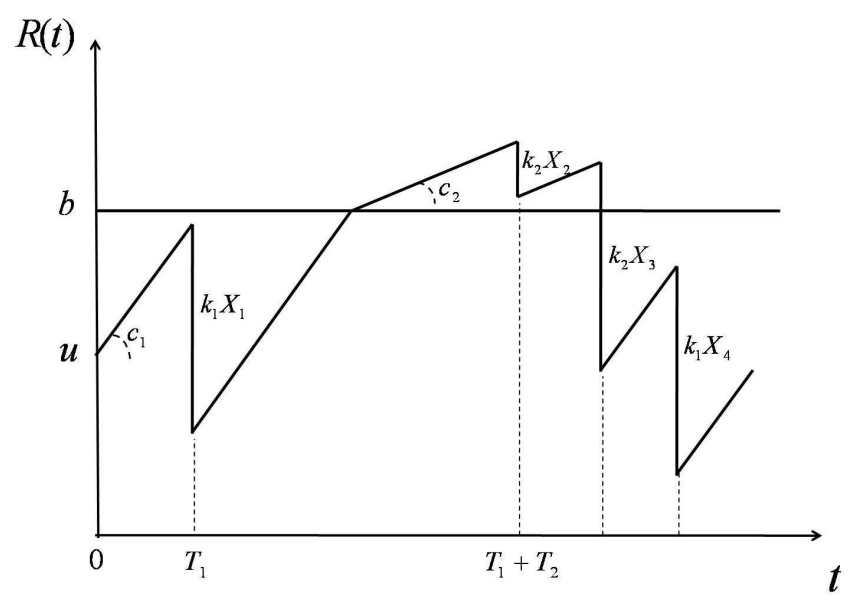

Fig. 1 Threshold reinsurance strategy

\section{Integro-differential equation for the Gerber-Shiu function}

In this section, we will give the integro-differential equations and boundary conditions satisfied by the Gerber-Shiu discounted penalty function. 1 .

Let $d \cdot / d u$ denote the differentiation operator with respect to $u$. Moreover, define $\prod_{j=2}^{1} \cdot=$

In a model with a threshold reinsurance strategy and with inter-occurrence times generalized Erlang $(n)$-distributed, the discounted penalty function $\phi(u)$ behaves differently, 
depending on whether its initial surplus $u$ is below or above the level $b$. Hence, for notational convenience, we write

$$
\phi(u)=\left\{\begin{array}{l}
\phi_{1}(u) \quad 0 \leq u<b \\
\phi_{2}(u) \quad u \geq b
\end{array} .\right.
$$

The following theorem provides integro-differential equations for the function $\phi(u)$.

Theorem 1 The integro-differential equations for the Gerber-Shiu discounted penalty function defined in (3), in a model with a threshold reinsurance strategy with inter-occurrence times generalized Erlang(n)-distributed with parameters $\lambda_{1}, \ldots, \lambda_{n}$, taking into account (4) is, for $0<u<b$,

$$
\begin{gathered}
\left(\prod_{j=1}^{n}\left(\delta+\lambda_{j}-c_{1} \frac{d \cdot}{d u}\right)\right) \phi_{1}(u)-\left(\prod_{j=1}^{n} \lambda_{j}\right) \int_{0}^{\frac{u}{k_{1}}} \phi_{1}\left(u-x k_{1}\right) d F(x) \\
-\left(\prod_{j=1}^{n} \lambda_{j}\right) \int_{\frac{u}{k_{1}}}^{\infty} w\left(u, x k_{1}-u\right) d F(x)=0
\end{gathered}
$$

and for $u>b$

$$
\begin{gathered}
\left(\prod_{j=1}^{n}\left(\delta+\lambda_{j}-c_{2} \frac{d \cdot}{d u}\right)\right) \phi_{2}(u)-\left(\prod_{j=1}^{n} \lambda_{j}\right) \int_{0}^{\frac{u-b}{k_{2}}} \phi_{2}\left(u-x k_{2}\right) d F(x) \\
-\left(\prod_{j=1}^{n} \lambda_{j}\right) \int_{\frac{u-b}{k_{2}}}^{\frac{u}{k_{2}}} \phi_{1}\left(u-x k_{2}\right) d F(x) \\
-\left(\prod_{j=1}^{n} \lambda_{j}\right) \int_{\frac{u}{k_{2}}}^{\infty} w\left(u, x k_{2}-u\right) d F(x)=0,
\end{gathered}
$$

with boundary conditions,

$$
\left.\phi_{1}(u)\right|_{u=b^{-}}=\phi_{2}(b) \text {, }
$$

and for $k=1, \ldots, n$

$$
\begin{gathered}
\left.c_{1}\left(\prod_{j=2}^{k}\left(\delta+\lambda_{j-1}-c_{1} \frac{d \cdot}{d u}\right)\right) \frac{d \phi_{1}(u)}{d u}\right|_{u=b}+\left(\prod_{s=1}^{n} \lambda_{s}\right) a_{k} \int_{0}^{\frac{b}{k_{1}}} \phi_{1}\left(b-x k_{1}\right) d F(x) \\
+\left(\prod_{s=1}^{n} \lambda_{s}\right) a_{k} \int_{\frac{b}{k_{1}}}^{\infty} w\left(b, x k_{1}-b\right) d F(x) \\
=\left.c_{2}\left(\prod_{j=2}^{k}\left(\delta+\lambda_{j-1}-c_{2} \frac{d \cdot}{d u}\right)\right) \frac{d \phi_{2}(u)}{d u}\right|_{u=b}+\left(\prod_{s=1}^{n} \lambda_{s}\right) a_{k} \int_{0}^{\frac{b}{k_{2}}} \phi_{1}\left(b-x k_{2}\right) d F(x) \\
+\left(\prod_{s=1}^{n} \lambda_{s}\right) a_{k} \int_{\frac{b}{k_{2}}}^{\infty} w\left(b, x k_{2}-b\right) d F(x),
\end{gathered}
$$

being

$$
a_{k}=\left\{\begin{array}{ll}
0 & k=1, \ldots, n-1 \\
1 & k=n
\end{array} .\right.
$$

Proof The proof, which follows Albrecher et al. (2005), is included in Appendix A. 
If the inter-occurrence time follows an exponential distribution with parameter $\lambda$, we obtain the following integro-differential equations and boundary conditions, for $0<u<b$

$$
\phi_{1}^{\prime}(u)=\frac{\lambda+\delta}{c_{1}} \phi_{1}(u)-\frac{\lambda}{c_{1}} \int_{0}^{\frac{u}{k_{1}}} \phi_{1}\left(u-x k_{1}\right) d F(x)-\frac{\lambda}{c_{1}} \xi_{1}(u),
$$

and for $u>b$

$$
\begin{aligned}
\phi_{2}^{\prime}(u)= & \frac{\lambda+\delta}{c_{2}} \phi_{2}(u)-\frac{\lambda}{c_{2}}\left[\int_{0}^{\frac{u-b}{k_{2}}} \phi_{2}\left(u-x k_{2}\right) d F(x)\right. \\
& \left.+\int_{\frac{u-b}{k_{2}}}^{\frac{u}{k_{2}}} \phi_{1}\left(u-x k_{2}\right) d F(x)\right]-\frac{\lambda}{c_{2}} \xi_{2}(u),
\end{aligned}
$$

with

$$
\xi_{i}(t)=\int_{\frac{t}{k_{i}}}^{\infty} w\left(t, x k_{i}-t\right) d F(x), i=1,2,
$$

with boundary conditions,

$$
\left.\phi_{1}(u)\right|_{u=b^{-}}=\phi_{2}(b),
$$

and

$$
\begin{aligned}
& c_{1} \phi_{1}^{\prime}(b)+\lambda \int_{0}^{\frac{b}{k_{1}}} \phi_{1}\left(b-x k_{1}\right) d F(x)+\lambda \int_{\frac{b}{k_{1}}}^{\infty} w\left(b, x k_{1}-b\right) d F(x) \\
= & c_{2} \phi_{2}^{\prime}(b)+\lambda \int_{0}^{\frac{b}{k_{2}}} \phi_{1}\left(b-x k_{2}\right) d F(x)+\lambda \int_{\frac{b}{k_{2}}}^{\infty} w\left(b, x k_{2}-b\right) d F(x) .
\end{aligned}
$$

Expressions (8) and (9) can be obtained by replacing the claim size random variable in Theorem 3.1. in Lin and Pavlova (2006) with $x k_{1}$ for $0<u<b$ and with $x k_{2}$ for $u>b$.

In order to obtain the time of ruin and ruin probability, in the following sections let $w(x, y)=1$ in (8), (9), and (10)

$$
\begin{array}{rlrl}
\phi_{1}^{\prime}(u)=\frac{\lambda+\delta}{c_{1}} & \phi_{1}(u)-\frac{\lambda}{c_{1}} \int_{0}^{\frac{u}{k_{1}}} \phi_{1}\left(u-x k_{1}\right) d F(x)-\frac{\lambda}{c_{1}}\left[1-F\left(\frac{u}{k_{1}}\right)\right], & 0<u<b, \\
\phi_{2}^{\prime}(u)= & \frac{\lambda+\delta}{c_{2}} \phi_{2}(u)-\frac{\lambda}{c_{2}}\left[\int_{0}^{\frac{u-b}{k_{2}}} \phi_{2}\left(u-x k_{2}\right) d F(x)\right. \\
& \left.+\int_{\frac{u-b}{k_{2}}}^{\frac{u}{k_{2}}} \phi_{1}\left(u-x k_{2}\right) d F(x)\right]-\frac{\lambda}{c_{2}}\left[1-F\left(\frac{u}{k_{2}}\right)\right], & u>b .
\end{array}
$$

with boundary conditions,

$$
\left.\phi_{1}(u)\right|_{u=b^{-}}=\phi_{2}(b),
$$

and

$$
\begin{aligned}
& c_{1} \phi_{1}^{\prime}(b)+\lambda \int_{0}^{\frac{b}{k_{1}}} \phi_{1}\left(b-x k_{1}\right) d F(x)+\lambda\left[1-F\left(\frac{b}{k_{1}}\right)\right] \\
= & c_{2} \phi_{2}^{\prime}(b)+\lambda \int_{0}^{\frac{b}{k_{2}}} \phi_{1}\left(b-x k_{2}\right) d F(x)+\lambda\left[1-F\left(\frac{b}{k_{2}}\right)\right] .
\end{aligned}
$$




\section{Ruin probability and time of ruin with individual claim amount Phase-type(N)}

In this section we obtain the differential equations for the ruin probability and for the time of ruin if ruin occurs, assuming that the individual claim amount is distributed as a phasetype $(N)$. Consider that $f(x)$ is the density function of a phase-type $(N)$ distribution satisfying the differential equation of order $N$

$$
\sum_{i=0}^{N} b_{i} f^{(i)}(x)=0
$$

with $b_{0}=1, b_{i}, i \geq 1, \ldots, N \in \Re, f^{(i)}(x)$ the $i$-th derivative of $f(x)$, being $f^{(0)}(x)=f(x)$ (Hipp 2006).

From (11) and (12), differentiating $N$ times with respect to $u$, the ordinal differential equations of order $N+1$ are obtained

Theorem 2 The ordinary differential equations for the Gerber-Shiu discounted penalty function defined in (3) in a model with a threshold reinsurance strategy with inter-occurrence time following an exponential distribution with parameter $\lambda$ and individual claim amounts phase-type $(N)$ with density satisfying (14), taking into account (4) are,

$$
\begin{aligned}
\phi_{i}^{(N+1)}(u)= & \left(\frac{\delta}{c_{i} k_{i}^{N} b_{N}}\right) \phi_{i}(u)+\left(\frac{\lambda+\delta}{c_{i}}-\frac{b_{N-1}}{k_{i} b_{N}}\right) \phi_{i}^{(N)}(u) \\
& -\sum_{s=1}^{N-1} \frac{1}{k_{i}^{N-s}}\left(\frac{\lambda}{c_{i}} f^{(N-1-s)}(0)+\frac{b_{s-1}}{k_{i} b_{N}}-\frac{(\lambda+\delta) b_{s}}{c_{i} b_{N}}\right. \\
& \left.+\frac{\lambda}{c_{i} b_{N}} \sum_{h=s+1}^{N-1} b_{h} f^{(h-s-1)}(0)\right) \phi_{i}^{(s)}(u),
\end{aligned}
$$

where $\phi_{i}(u), i=1,2$ being $i=1$ for $0<u<b$ and $i=2$ for $u>b$.

Proof The proof is included in Appendix B.

As a particular case, from (15), it is possible to obtain the equations if the individual claim amount is a unitary exponential, knowing that it is a phase-type(1) distribution with parameter $b_{1}=1$,

$$
\phi_{i}^{\prime \prime}(u)-\left(\frac{\lambda+\delta}{c_{i}}-\frac{1}{k_{i}}\right) \phi_{i}^{\prime}(u)-\frac{\delta}{c_{i} k_{i}} \phi_{i}(u)=0
$$

and if the individual claim amount is distributed as a phase-type(2),

$$
\begin{aligned}
\phi_{i}^{\prime \prime \prime}(u)= & \left(\frac{\lambda+\delta}{c_{i}}-\frac{b_{1}}{k_{i} b_{2}}\right) \phi_{i}^{\prime \prime}(u)+\left(\frac{b_{1}(\lambda+\delta)}{c_{i} k_{i} b_{2}}-\frac{1}{k_{i}^{2} b_{2}}-\frac{\lambda}{k_{i} c_{i}} f(0)\right) \phi_{i}^{\prime}(u) \\
& +\frac{\delta}{b_{2} k_{i}^{2} c_{i}} \phi_{i}(u)
\end{aligned}
$$

with $i=1$ for $0<u<b$ and $i=2$ for $u>b$. 
4.1 Ruin probability and time of ruin with individual claim amount exponential

In this subsection we consider the case when the individual claim amount is distributed as an exponential with unitary mean, $f(x)=e^{-x}$.

From (16), it is easy to obtain the characteristic equations,

$$
\begin{aligned}
& r^{2}-\left(\frac{\lambda+\delta}{c_{1}}-\frac{1}{k_{1}}\right) r-\frac{\delta}{c_{1} k_{1}}=0, \quad 0 \leq u<b, \\
& s^{2}-\left(\frac{\lambda+\delta}{c_{2}}-\frac{1}{k_{2}}\right) s-\frac{\delta}{c_{2} k_{2}}=0, \quad u \geq b,
\end{aligned}
$$

with $r_{1}<0, r_{2} \geq 0, s_{1}<0$ and $s_{2} \geq 0$ the real roots of the characteristic equations. The roots $r_{2}$ and $s_{2}$ are equal to zero if $\delta=0$ (the ruin probability case), and positive if $\delta>0$ (the defective LT of the time of ruin).

Then the defective LT of the time of ruin $\phi(u)=E\left[e^{-\delta T} I(T<\infty) \mid R(0)=u\right]$ is

$$
\phi(u)=\left\{\begin{array}{cr}
\phi_{1}(u)=C_{1} e^{r_{1} u}+C_{2} e^{r_{2} u}, \quad 0 \leq u<b \\
\phi_{2}(u)=D_{1} e^{s_{1} u}+D_{2} e^{s_{2} u}, & u \geq b
\end{array} .\right.
$$

From the condition $\lim _{u \rightarrow \infty} \phi(u)=0$, we know that $D_{2}=0$, from the continuity condition $\left.\phi_{1}(u)\right|_{u=b^{-}}=\phi_{2}(b)$ we obtain $\sum_{i=1}^{2} C_{i} e^{r_{i} b}-D_{1} e^{s_{1} b}=0$, and substituting (19) in (13) and rearranging terms, we obtain two additional conditions, $\sum_{i=1}^{2} \frac{C_{i}}{k_{1} r_{i}+1}=1$ and $\sum_{i=1}^{2} \frac{C_{i}}{k_{2} r_{i}+1}(1-$ $\left.e^{b\left(r_{i}+\frac{1}{k_{2}}\right)}\right)+\frac{D_{1}}{s_{1} k_{2}+1} e^{b\left(s_{1}+\frac{1}{k_{2}}\right)}=1$, which allow us to obtain the coefficients $C_{i}, i=1,2$ and $D_{1}$. So,

$$
\begin{aligned}
& C_{1}(\delta)=\frac{a_{2,1} a_{1,1}\left(\left(k_{2} s_{1}+1\right) r_{2}\left(k_{2}-k_{1}\right)-a_{1,2} k_{2}\left(r_{2}-s_{1}\right) e^{\frac{a_{2,2}}{k_{2}} b}\right)}{\left(k_{2} s_{1}+1\right)\left(r_{1}-r_{2}\right)\left(k_{1}-k_{2}\right)-k_{2}\left(a_{1,1} a_{2,2}\left(s_{1}-r_{1}\right) e^{\frac{a_{2,1}}{k_{2}} b}-a_{1,2} a_{2,1}\left(s_{1}-r_{2}\right) e^{\frac{a_{2,2}}{k_{2}}}\right)}, \\
& C_{2}(\delta)=a_{1,2}-\frac{a_{1,2}}{a_{1,1}} C_{1}(\delta), \\
& D_{1}(\delta)=a_{1,2} e^{\left(r_{2}-s_{1}\right) b}+\left(e^{\left(r_{1}-s_{1}\right) b}-\frac{a_{1,2}}{a_{1,1}} e^{\left(r_{2}-s_{1}\right) b}\right) C_{1}(\delta),
\end{aligned}
$$

where $a_{i, j}=\left(k_{i} r_{j}+1\right), i, j=1,2$. then,

To obtain the ruin probability, $\phi(u)=E[I(T<\infty) \mid R(0)=u]=\psi(u)$, let $\delta=0$ in (19),

$$
\psi(u)=\left\{\begin{array}{lr}
\psi_{1}(u)=1-\left(1+\rho_{1}\right) C_{1}(0)+C_{1}(0) e^{-\frac{\rho_{1}}{k_{1}\left(1+\rho_{1}\right)} u}, 0 \leq u<b \\
\psi_{2}(u)=\psi_{1}(b) e^{\frac{\rho_{2}}{k_{2}\left(1+\rho_{2}\right)}(b-u)}, & u \geq b
\end{array}\right.
$$

where

$$
C_{1}(0)=\frac{h}{h\left(1+\rho_{1}\right)+\left(k_{1}-k_{2}\right) \rho_{1}\left(1+\rho_{1}\right) e^{-\frac{b}{k_{2}}}+\left(k_{2} \rho_{1}-h\right) e^{-\frac{\rho_{1}}{k_{1}\left(1+\rho_{1}\right)} b}},
$$

with $h=\left(k_{1}+\rho_{1}\left(k_{1}-k_{2}\right)\right) \rho_{2}$.

From (19) and (20), it is easy to obtain the moments of the time of ruin. For example, the expected time of ruin if ruin occurs is given by

$$
E[T \mid T<\infty]=-\frac{\left.\frac{\partial \phi(u)}{\partial \delta}\right|_{\delta=0^{+}}}{\psi(u)} .
$$


Then for $0 \leq u<b$,

$$
E[T \mid T<\infty]=-\frac{\left.\frac{\partial C_{1}(\delta)}{\partial \delta}\right|_{\delta=0^{+}} e^{-\frac{\rho_{1}}{k_{1}\left(1+\rho_{1}\right)} u}-\frac{C_{1}(0) u e^{-\frac{\rho_{1}}{k_{1}\left(1+\rho_{1}\right)}}}{\lambda k_{1} \rho_{1}\left(1+\rho_{1}\right)}+\left.\frac{\partial C_{2}(\delta)}{\partial \delta}\right|_{\delta=0^{+}}+\frac{C_{2}(0) u}{\lambda k_{1} \rho_{1}}}{1-\left(1+\rho_{1}\right) C_{1}(0)+C_{1}(0) e^{-\frac{\rho_{1}}{k_{1}\left(1+\rho_{1}\right)}} u},
$$

and for $u \geq b$,

$$
E[T \mid T<\infty]=-\frac{\left.\frac{\partial D_{1}(\delta)}{\partial \delta}\right|_{\delta=0^{+}}}{D_{1}(0)}+\frac{1}{\lambda k_{2} \rho_{2}\left(1+\rho_{2}\right)} u .
$$

We can observe that for $u \geq b$ the expression obtained for $E[T \mid T<\infty]$ is a first degree polynomial on $u$, similar to the model without reinsurance (see Gerber 1979).

The variance is

$$
V[T \mid T<\infty]=E\left[T^{2} \mid T<\infty\right]-(E[T \mid T<\infty])^{2} .
$$

For $0 \leq u<b$

$$
E\left[T^{2} \mid T<\infty\right]=\frac{E\left[T^{2} I(T<\infty)\right]}{1-\left(1+\rho_{1}\right) C_{1}(0)+C_{1}(0) e^{-\frac{\rho_{1}}{k_{1}\left(1+\rho_{1}\right)} u}},
$$

being

$$
\begin{aligned}
E\left[T^{2} I(T<\infty)\right]= & \left.\frac{\partial^{2} C_{1}(\delta)}{\partial \delta^{2}}\right|_{\delta=0^{+}} e^{-\frac{\rho_{1}}{k_{1}\left(1+\rho_{1}\right)} u}-\left.2 \frac{\partial C_{1}(\delta)}{\partial \delta}\right|_{\delta=0^{+}} \frac{u e^{-\frac{\rho_{1}}{k_{1}\left(1+\rho_{1}\right)}} u}{\lambda k_{1} \rho_{1}\left(1+\rho_{1}\right)} \\
& +C_{1}(0) u e^{-\frac{\rho_{1}}{k_{1}\left(1+\rho_{1}\right)} u}\left(\frac{u}{\left(\lambda k_{1} \rho_{1}\left(1+\rho_{1}\right)\right)^{2}}+\frac{2}{\lambda^{2} k_{1} \rho_{1}^{3}}\right) \\
& +\left.\frac{\partial^{2} C_{2}(\delta)}{\partial \delta^{2}}\right|_{\delta=0^{+}}+\left.2 \frac{\partial C_{2}(\delta)}{\partial \delta}\right|_{\delta=0^{+}} \frac{u}{\lambda k_{1} \rho_{1}} \\
& +C_{2}(0) u\left(\frac{u}{\left(\lambda k_{1} \rho_{1}\right)^{2}}-\frac{2}{\lambda^{2} k_{1} \rho_{1}^{3}}\right)
\end{aligned}
$$

For $u \geq b$,

$$
\begin{aligned}
E\left[T^{2} \mid T<\infty\right]= & \frac{\left.\frac{\partial^{2} D_{1}(\delta)}{\partial \delta^{2}}\right|_{\delta=0^{+}}}{D_{1}(0)}+2\left(\frac{1}{\lambda^{2} k_{2} \rho_{2}^{3}}-\frac{\left.\frac{\partial D_{1}(\delta)}{\partial \delta}\right|_{\delta=0^{+}}}{D_{1}(0) \lambda k_{2} \rho_{2}\left(1+\rho_{2}\right)}\right) u+ \\
& \frac{1}{\left(\lambda k_{2} \rho_{2}\left(1+\rho_{2}\right)\right)^{2}} u^{2}
\end{aligned}
$$

being

$$
V[T \mid T<\infty]=\frac{\left.\frac{\partial^{2} D_{1}(\delta)}{\partial \delta^{2}}\right|_{\delta=0^{+}}}{D_{1}(0)}-\left(\frac{\left.\frac{\partial D_{1}(\delta)}{\partial \delta}\right|_{\delta=0^{+}}}{D_{1}(0)}\right)^{2}+\frac{2}{\lambda^{2} k_{2} \rho_{2}^{3}} u .
$$

From equations (21) to (27) we can obtain the expressions in a model with proportional reinsurance (for $k_{1}=k_{2}=k$ ) and in a model without reinsurance (for $k_{1}=k_{2}=1$ ). 
Then, if we let $k_{1}=k_{2}=k$, the following expressions are obtained

$$
\begin{gathered}
E[T \mid T<\infty]=\frac{1}{\lambda \rho_{N}}+\frac{1}{\lambda k \rho_{N}\left(1+\rho_{N}\right)} u, \\
V[T \mid T<\infty]=\frac{2+\rho_{N}}{\lambda^{2} \rho_{N}^{3}}+\frac{2}{\lambda^{2} k \rho_{N}^{3}} u .
\end{gathered}
$$

And for $k_{1}=k_{2}=1$,

$$
\begin{gathered}
E[T \mid T<\infty]=\frac{1}{\lambda \rho}+\frac{1}{\lambda \rho(1+\rho)} u, \\
V[T \mid T<\infty]=\frac{2+\rho}{\lambda^{2} \rho^{3}}+\frac{2}{\lambda^{2} \rho^{3}} u,
\end{gathered}
$$

expressions that can also be found in Dickson (2005), p.188.

Example 1 Let $\rho=0.15, \rho_{R}=0.25, \lambda=1, \delta=0.03$ and $b=8$, the limits for the retention levels necessary to meet the "net profit" condition being $0.4<k_{1} \leq 1$ and $0.4<k_{2} \leq 1$. The values chosen are $k_{1}=0.8, k_{2}=0.45$, with $k_{1}>k_{2}$ since, as we will see later, if we consider the probability of ruin as a criterion of decision, in the optimal combinations of $k_{1}$ and $k_{2}$, the retention level when the reserves are below the threshold is always higher than the retention level when the reserves are above the threshold.

The defective LT of the time of ruin is,

$$
\phi(u)=\left\{\begin{array}{lr}
\phi_{1}(u)=0.7940 e^{-0.2636 u}-0.0070 e^{0.1580 u}, 0 \leq u<8, \\
\phi_{2}(u)=1.4625 e^{-0.3772 u,} \quad u \geq 8,
\end{array}\right.
$$

and the expression for the probability of ruin,

$$
\psi(u)=\left\{\begin{array}{lr}
\psi_{1}(u)=0.2906+0.6305 e^{-0.1388 u}, 0 \leq u<8, \\
\psi_{2}(u)=0.8054 e^{-0.06006 u}, & u \geq 8
\end{array}\right.
$$

Table 1 shows the results obtained for the probability of ruin, the defective LT of the time of ruin and the expectation, the variance and the variation coefficient of the moment of ruin, for different values of $u$,

Table $1 \phi(u), \psi(u), E[T \mid T<\infty], V[T \mid T<\infty]$ and $C_{V}$ for $X \sim \operatorname{Exp}(1)$

\begin{tabular}{llllll}
\hline$u$ & $\phi(u)$ & $\psi(u)$ & $E[T \mid T<\infty]$ & $V[T \mid T<\infty]$ & $C_{V}=\frac{\sqrt{V[T \mid T<\infty]}}{E[T \mid T<\infty]}$ \\
\hline 0 & 0.7870 & 0.9211 & 65.00 & 230297 & 7.38 \\
4 & 0.2634 & 0.6524 & 389.17 & $1.30 \times 10^{6}$ & 2.93 \\
8 & 0.0715 & 0.4981 & 712.12 & $2.22 \times 10^{6}$ & 2.09 \\
12 & 0.0158 & 0.3917 & 1023.47 & $3.05 \times 10^{6}$ & 1.70 \\
16 & 0.0034 & 0.3081 & 1334.83 & $3.88 \times 10^{6}$ & 1.47 \\
20 & 0.0007 & 0.2423 & 1646.18 & $4.71 \times 10^{6}$ & 1.31 \\
\hline
\end{tabular}


4.2 Ruin probability and time of ruin with individual claim amount Phase-type(2)

From (17), the characteristic equation for $0 \leq u<b$

$$
r^{3}-\left(\frac{\lambda+\delta}{c_{1}}-\frac{b_{1}}{k_{1} b_{2}}\right) r^{2}-\left(\frac{b_{1}(\lambda+\delta)}{c_{1} k_{1} b_{2}}-\frac{1}{k_{1}^{2} b_{2}}-\frac{\lambda}{k_{1} c_{1}} f(0)\right) r-\frac{\delta}{b_{2} k_{1}^{2} c_{1}}=0,
$$

and for $u \geq b$,

$$
s^{3}-\left(\frac{\lambda+\delta}{c_{2}}-\frac{b_{1}}{k_{2} b_{2}}\right) s^{2}-\left(\frac{b_{1}(\lambda+\delta)}{c_{2} k_{2} b_{2}}-\frac{1}{k_{2}^{2} b_{2}}-\frac{\lambda}{k_{2} c_{2}} f(0)\right) s-\frac{\delta}{b_{2} k_{2}^{2} c_{2}}=0,
$$

with $r_{i}, s_{i}, i=1,2,3$ the real and distinct roots of the characteristic equations. Then

$$
\phi(u)=\left\{\begin{array}{l}
\phi_{1}(u)=\sum_{i=1}^{3} F_{i} e^{r_{i} u}, 0 \leq u<b, \\
\phi_{2}(u)=\sum_{i=1}^{3} G_{i} e^{s_{i} u}, \quad u \geq b .
\end{array}\right.
$$

In order to obtain these coefficients, six equations are needed. The first one is obtained from the condition $\lim _{u \longrightarrow \infty} \phi(u)=0$. The second can be obtained considering that $\phi(u)$ must be continuous, $\left.\phi_{1}(u)\right|_{u=b^{-}}=\phi_{2}(b)$. The other four are obtained substituting (30) in (13) and rearranging terms. To obtain the ruin probability, we have to consider $\delta=0$, so $r_{3}=s_{3}=0$.

Example 2 We analyze the particular case $\operatorname{Erlang}(2, \beta)$, i.e. $f(x)=\beta^{2} x e^{-\beta x}$. The Erlang $(2, \beta)$ distribution is a phase-type(2) distribution with $b_{1}=2 / \beta$ and $b_{2}=1 / \beta^{2}$ (Dickson and Drekic 2004). Then, the characteristic equations are

$$
\begin{array}{rrr}
r^{3}+\left(\frac{2 \beta}{k_{1}}-\frac{\lambda+\delta}{c_{1}}\right) r^{2}+\left(\frac{\beta^{2}}{k_{1}^{2}}-\frac{2 \beta(\lambda+\delta)}{c_{1} k_{1}}\right) r-\frac{\delta \beta^{2}}{c_{1} k_{1}^{2}}=0, & 0 \leq u<b, \\
s^{3}+\left(\frac{2 \beta}{k_{2}}-\frac{\lambda+\delta}{c_{2}}\right) s^{2}+\left(\frac{\beta^{2}}{k_{2}^{2}}-\frac{2 \beta(\lambda+\delta)}{c_{2} k_{2}}\right) s-\frac{\delta \beta^{2}}{c_{2} k_{2}^{2}}=0, & u \geq b .
\end{array}
$$

It is easy to demonstrate that two of the roots are negative $\left(r_{i}, s_{i}<0, i=1,2\right)$ and that $r_{3}$, $s_{3}>0$ if $\delta>0$ or $r_{3}, s_{3}=0$ if $\delta=0$. The system of equations that we need to find the coefficients is

$$
\left\{\begin{array}{l}
G_{3}=0 \\
\sum_{i=1}^{3} F_{i} e^{r_{i} b}-\sum_{i=1}^{2} G_{i} e^{s_{i} b}=0 \\
\sum_{i=1}^{3} \frac{F_{i}}{r_{i} k_{1}+\beta}=\frac{1}{\beta} \\
\sum_{i=1}^{3} \frac{F_{i}}{\left(r_{i} k_{1}+\beta\right)^{2}}=\frac{1}{\beta^{2}} \\
\sum_{i=1}^{3} \frac{F_{i}}{k_{2} r_{i}+\beta}\left(1-e^{b\left(r_{i}+\frac{\beta}{k_{2}}\right)}\right)+\sum_{i=1}^{2} \frac{G_{i} e^{b\left(s_{i}+\frac{\beta}{k_{2}}\right)}}{k_{2} s_{i}+\beta}=\frac{1}{\beta} \\
\sum_{i=1}^{3} \frac{F_{i}\left(e^{b\left(r_{i}+\frac{\beta}{k_{2}}\right)}\left(b\left(k_{2} r_{i}+\beta\right)-k_{2}\right)+k_{2}\right)}{\left(k_{2} r_{i}+\beta\right)^{2}}-\sum_{i=1}^{2} \frac{G_{i} e^{b\left(s_{i}+\frac{\beta}{k_{2}}\right)}}{\left(b\left(k_{2} s_{i}+\beta\right)-k_{2}\right)}=\frac{k_{2}}{\beta^{2}}
\end{array}\right.
$$

To obtain the ruin probability, the six equations to obtain the coefficients are (31), with $\delta=r_{3}=s_{3}=0$. 
Notation: As we mentioned above, the expressions in the model with a proportional reinsurance can be obtained as a particular case of the threshold reinsurance. So, when the claim size distribution is $\operatorname{Erlang}(2, \beta)$, from the example, if we let $k_{1}=k_{2}=k$ the defective LT of the time of ruin is

$$
\phi(u)=-\frac{r_{2}\left(k r_{1}+\beta\right)^{2}}{\left(r_{1}-r_{2}\right) \beta^{2}} e^{r_{1} u}+\frac{r_{1}\left(k r_{2}+\beta\right)^{2}}{\left(r_{1}-r_{2}\right) \beta^{2}} e^{r_{2} u}, \quad u \geq 0,
$$

$r_{1}$ and $r_{2}$ being the negative roots of the equation

$$
r^{3}+\left(\frac{2 \beta}{k}-\frac{\lambda+\delta}{c}\right) r^{2}+\left(\frac{\beta^{2}}{k^{2}}-\frac{2 \beta(\lambda+\delta)}{c k}\right) r-\frac{\delta \beta^{2}}{c k^{2}}=0 .
$$

And the ruin probability is

$$
\begin{aligned}
\psi(u)= & \frac{3+2 \rho_{N}+\sqrt{9+8 \rho_{N}}}{2\left(1+\rho_{N}\right) \sqrt{9+8 \rho_{N}}} \exp \left(\frac{\left(-3-4 \rho_{N}+\sqrt{9+8 \rho_{N}}\right) \beta}{4 k\left(1+\rho_{N}\right)} u\right) \\
& +\frac{\sqrt{9+8 \rho_{N}}-3-2 \rho_{N}}{2\left(1+\rho_{N}\right) \sqrt{9+8 \rho_{N}}} \exp \left(-\frac{\left(3+4 \rho_{N}+\sqrt{9+8 \rho_{N}}\right) \beta}{4 k\left(1+\rho_{N}\right)} u\right), \quad u \geq 0 .
\end{aligned}
$$

Let $k_{1}=k_{2}=1$; then, the defective LT of the time of ruin in a model without reinsurance is obtained,

$$
\phi(u)=-\frac{r_{2}\left(r_{1}+\beta\right)^{2}}{\left(r_{1}-r_{2}\right) \beta^{2}} e^{r_{1} u}+\frac{r_{1}\left(r_{2}+\beta\right)^{2}}{\left(r_{1}-r_{2}\right) \beta^{2}} e^{r_{2} u}, \quad u \geq 0,
$$

$r_{1}$ and $r_{2}$ being the negative roots of the equation

$$
r^{3}+\left(2 \beta-\frac{\lambda+\delta}{c}\right) r^{2}+\left(\beta^{2}-\frac{2 \beta(\lambda+\delta)}{c}\right) r-\frac{\delta \beta^{2}}{c}=0
$$

And the ruin probability,

$$
\begin{aligned}
\psi(u)= & \frac{3+2 \rho+\sqrt{9+8 \rho}}{2(1+\rho) \sqrt{9+8 \rho}} e^{\frac{(-3-4 \rho+\sqrt{9+8 \rho}) \beta}{4(1+\rho)} u} \\
& +\frac{\sqrt{9+8 \rho}-3-2 \rho}{2(1+\rho) \sqrt{9+8 \rho}} e^{-\frac{(3+4 \rho+\sqrt{9+8 \rho}) \beta}{4(1+\rho)} u}, \quad u \geq 0 .
\end{aligned}
$$

Example 3 We perform a numerical application with the same values used in the case of the unitary exponential amount: $\rho=0.15, \rho_{R}=0.25, \lambda=1, k_{1}=0.8, k_{2}=0.45, \delta=0.03$, $\beta=2$ and $b=8$.

The mean claim amount for the distribution $\operatorname{Erlang}(2,2)$ is 1 , as in the numerical application of the exponential. We analyze this case, to see whether the change in the distribution of the claim amount has any significant effect on the behaviour of the magnitudes, even if the mean claim is not altered.

The defective LT of the time of ruin is

$$
\phi(u)=\left\{\begin{array}{l}
\phi_{1}(u)=-0.0225 e^{-3.6975 u}+0.8303 e^{-0.3291 u}-0.0034 e^{0.1711 u}, 0 \leq u<8, \\
\phi_{2}(u)=-2.85 \times 10^{18} e^{-6.6392 u}+1.6969 e^{-0.4507 u}, \quad u \geq 8 .
\end{array}\right.
$$


And the ruin probability,

$$
\psi(u)=\left\{\begin{array}{l}
\psi_{1}(u)=-0.0095 e^{-3.7012 u}+0.7017 e^{-0.1876 u}+0.2213,0 \leq u<8 \\
\psi_{2}(u)=2.38 \times 10^{18} e^{-6.6464 u}+0.7182 e^{-0.0803 u}, \quad u \geq 8
\end{array}\right.
$$

Table 2 shows the results obtained for the probability of ruin, the defective LT of the time of ruin, the expectation, the variance and the variation coefficient of the moment of ruin for different values of $u$,

Table $2 \phi(u), \psi(u), E[T \mid T<\infty], V[T \mid T<\infty]$ and $C_{V}$ for $X \sim \operatorname{Erlang}(2,2)$

\begin{tabular}{llllll}
\hline$u$ & $\phi(u)$ & $\psi(u)$ & $E[T \mid T<\infty]$ & $V[T \mid T<\infty]$ & $C_{V}=\frac{\sqrt{V[T \mid T<\infty]}}{E[T \mid T<\infty]}$ \\
\hline 0 & 0.8043 & 0.9134 & 42.88 & 120387 & 8.09 \\
4 & 0.2157 & 0.5526 & 346.48 & 918753 & 2.76 \\
8 & 0.0460 & 0.3777 & 673.65 & $1.63 \times 10^{6}$ & 1.89 \\
12 & 0.0075 & 0.2739 & 985.99 & $2.25 \times 10^{6}$ & 1.52 \\
16 & 0.0012 & 0.1986 & 1298.30 & $2.88 \times 10^{6}$ & 1.30 \\
20 & 0.0002 & 0.1440 & 1610.61 & $3.50 \times 10^{6}$ & 1.16 \\
\hline
\end{tabular}

Comparing the results obtained in Table 2 with those in Table 1, the change in the distribution of the amount of the claims does not significantly alter the results of the magnitudes analyzed. Considering a distribution $\operatorname{Erlang}(2,2)$ the probabilities of ruin, expectation and the variance of the moment of ruin are lower than in the case of the amount $\operatorname{Exp}(1)$. Nonetheless, the behaviour with respect to the initial level of the reserves of different magnitudes is the same for the two distributions, i.e. when $u$ increases, the ruin probability and the variation coefficient decrease, and the expectation and variance of the moment of ruin increase.

\section{Comparison of reinsurance strategies}

In this last section we present a series of numerical and comparative analyzes of the new threshold proportional reinsurance strategy.

In Subsect. 5.1 we obtain the optimal threshold reinsurance strategy from the point of view of the probability of ruin if the individual claim amount is distributed as an exponential with unitary mean and an $\operatorname{Erlang}(2, \beta)$. In Subsect. 5.2, after obtaining the value of the percentage of retention that minimizes the probability of ruin in a model with proportional reinsurance, we compare the two reinsurance strategies, including the results for the exponential claim amounts and Erlang $(2, \beta)$, reaching the conclusion that the most interesting strategy for the insurer is the threshold strategy.

The fact that the threshold reinsurance strategy obtains lower probabilities of ruin means that this strategy emerges as a tool for the insurer to manage the initial investment that the portfolio requires. $\beta=2$.

The values of the parameters used in this section are $\lambda=1, \rho=0.15, \rho_{R}=0.25$ and 
5.1 Optimal strategy with threshold proportional reinsurance

The objective of this subsection is to find the optimal strategy for minimizing the probability of ruin. The insurer can decide on the decision variables. In the first option, the insurer sets the level of threshold $b$, and seeks the best combination of the retention percentages, $\left(k_{1}, k_{2}\right)$, which allows him to minimize this probability. In the second option, the decision variables are the threshold level and the percentages of retention, the optimal strategy being $\left(b, k_{1}, k_{2}\right)$; this allows us to obtain the minimal probability of ruin, which we will term $\psi_{\min }^{k_{1} \neq k_{2}}(u)$.

If we analyze the case $X \sim \operatorname{Exp}(1)$, first we set the threshold value $b$, obtaining the optimal combination $\left(k_{1}, k_{2}\right)$ that the insurer should apply to minimize the probability of ruin. Table 3 shows the results calculated with the function NMinimize of the software Mathematica for three different threshold levels,

Table 3 Optimal combinations $\left(k_{1}, k_{2}\right)$ for different values of $b$ if $X \sim \operatorname{Exp}(1)$

\begin{tabular}{|c|c|c|c|c|c|c|c|c|c|}
\hline \multirow[b]{2}{*}{$u$} & \multicolumn{3}{|c|}{$b=2$} & \multicolumn{3}{|l|}{$b=8$} & \multicolumn{3}{|l|}{$b=15$} \\
\hline & $k_{1}$ & $k_{2}$ & $\psi(u)$ & $k_{1}$ & $k_{2}$ & $\psi(u)$ & $k_{1}$ & $k_{2}$ & $\psi(u)$ \\
\hline 0 & 1 & 0.7806 & 0.8659 & 1 & 0.7602 & 0.8666 & 1 & 0.7603 & 0.8684 \\
\hline 4 & 1 & 0.7693 & 0.5001 & 1 & 0.7602 & 0.5053 & 0.8639 & 0.7584 & 0.5086 \\
\hline 8 & 1 & 0.7636 & 0.2865 & 0.91724 & 0.7590 & 0.2905 & 0.8105 & 0.7579 & 0.2923 \\
\hline 12 & 1 & 0.7616 & 0.1641 & 0.91738 & 0.7585 & 0.1664 & 0.7977 & 0.7578 & 0.1675 \\
\hline 16 & 1 & 0.7607 & 0.0939 & 0.91736 & 0.7583 & 0.0953 & 0.7963 & 0.7578 & 0.0959 \\
\hline 20 & 1 & 0.7601 & 0.0538 & 0.91735 & 0.7581 & 0.0545 & 0.7963 & 0.7578 & 0.0549 \\
\hline
\end{tabular}

We now consider the optimal strategy $\left(b, k_{1}, k_{2}\right)$ for obtaining $\psi_{\min }^{k_{1} \neq k_{2}}(u)$ if $X \sim \operatorname{Exp}(1)$. Table 4 presents the results obtained, and the expectation, the variance and the variation coefficient for different values of $u$,

Table 4 Optimal combination $\left(b, k_{1}, k_{2}\right)$ for different values of $u$ if $X \sim \operatorname{Exp}(1)$

\begin{tabular}{llllllll}
\hline$u$ & $b$ & $k_{1}$ & $k_{2}$ & $\psi_{\min }^{k_{1} \neq k_{2}}(u)$ & $E[T \mid T<\infty]$ & $V[T \mid T<\infty]$ & $C_{V}$ \\
\hline 0 & 3.2667 & 1 & 0.760031 & 0.864665 & 9.28326 & 1556.82 & 4.25029 \\
4 & 3.2675 & 1 & 0.759623 & 0.498067 & 47.2008 & 7781.53 & 1.86889 \\
8 & 3.2685 & 1 & 0.758708 & 0.285276 & 87.2039 & 14207.2 & 1.36684 \\
12 & 3.2692 & 1 & 0.758399 & 0.163396 & 127.205 & 20629 & 1.12911 \\
16 & 3.2689 & 1 & 0.758243 & 0.0935873 & 167.205 & 27049.9 & 0.983632 \\
20 & 3.2693 & 1 & 0.758149 & 0.0536035 & 207.206 & 33470.4 & 0.882936 \\
\hline
\end{tabular}

Therefore, the optimal strategy for the insurer is to choose a low threshold level (in this example approximately $b=3.27$ ), not to reinsure $\left(k_{1}=1\right)$ when the reserves are below this level and to reinsure with a retention level of approximately $76 \%$ when the reserves are above the threshold. The result obtained is consistent with the ones presented in Schmidli (2001, 2006).

Assuming that the individual claim amount follows an Erlang $(2,2)$, in Table 5 we present the optimal strategy $\left(b, k_{1}, k_{2}\right)$ for obtaining $\psi_{\min }^{k_{1} \neq k_{2}}(u)$. 
Table 5 Optimal combination $\left(b, k_{1}, k_{2}\right)$ for different values of $u$ if $X \sim \operatorname{Erlang}(2,2)$

\begin{tabular}{lllll}
\hline$u$ & $b$ & $k_{1}$ & $k_{2}$ & $\psi_{\min }^{k_{1} \neq k_{2}}(u)$ \\
\hline 0 & 1.98703 & 1 & 0.760973 & 0.864262 \\
4 & 1.9871 & 1 & 0.761572 & 0.415635 \\
8 & 1.98712 & 1 & 0.761895 & 0.195874 \\
12 & 1.98712 & 1 & 0.762009 & 0.0923087 \\
16 & 1.98721 & 1 & 0.762064 & 0.0435018 \\
20 & 1.98711 & 1 & 0.762098 & 0.0205009 \\
\hline
\end{tabular}

With $X \sim \operatorname{Erlang}(2,2)$, the optimal strategy for the insurer in order to minimize the probability of ruin is $\left(b, k_{1}, k_{2}\right) \approx(1.987,1,0.76)$.

\subsection{Comparison of proportional reinsurance strategies}

The aim of this subsection is to compare the threshold reinsurance strategy with the proportional reinsurance strategy from the point of view of the probability of ruin.

If $X \sim \operatorname{Exp}(1)$, we first show the values of the retention level, $k$, that make it possible to minimize the probability of ruin, and then compare the results with the optimal probabilities obtained in the above section with threshold reinsurance.

In a model with proportional reinsurance, Waters (1983) and Schmidli (2006) found the value of the retention percentage $k$ that maximizes the coefficient of fit as a way of minimizing the Lundberg bound for the probability of ruin. Therefore it is a value that does not depend on $u$, with

$$
k=\left(1-\frac{\rho}{\rho_{R}}\right)\left(1+\frac{1}{\sqrt{1+\rho_{R}}}\right) .
$$

Remembering that the probability of ruin in a model with proportional reinsurance is (Dickson 2005, p. 203),

$$
\psi(u)=\frac{k}{k\left(1+\rho_{R}\right)+\rho-\rho_{R}} e^{-\frac{\rho_{R}(k-1)+\rho}{k\left(\left(1+\rho_{R}\right) k+\rho-\rho_{R}\right)}} u
$$

Dickson and Waters (1996) obtain numerically the values of $k$ that minimize $\psi(u)$ for different values of $u$. However, it is easy to obtain the analytical formula for the retention level , $k_{o p}(u)$, that minimizes (33),

$$
k_{o p}(u)=\left\{\begin{array}{c}
\frac{-A^{2}+2 B A u+A \sqrt{A^{2}+4 B u^{2}}}{2 B\left(u \rho_{R}-A\right)} \text { if } u>\frac{(1+\rho) A}{\rho(2+\rho)-\rho_{R}}>0, \\
1 \quad \text { otherwise }
\end{array}\right.
$$

where $A=\left(\rho_{R}-\rho\right)$ and $B=\left(1+\rho_{R}\right)$.

The minimum values for the probability of ruin calculated with $k_{o p}(u)$ and $k=0.7577$, (obtained from (32)), are shown in Table 6, which shows the results for expectation, variance and the variation coefficient of the moment of ruin with $k_{o p}(u)$, 
Table 6 Minimum probabilities of ruin with proportional reinsurance with $k_{o p}(u)$ and $k$ if $X \sim \operatorname{Exp}(1)$

\begin{tabular}{lllllll}
\hline$u$ & $k_{o p}(u)$ & $\psi_{\min }^{k_{o p}}(u)$ & $\begin{array}{l}\psi_{\min }(u) \text { with } \\
k=0.7577\end{array}$ & $\begin{array}{l}E[T \mid T<\infty] \\
\text { with } k_{o p}(u)\end{array}$ & $\begin{array}{l}V[T \mid T<\infty] \\
\text { with } k_{o p}(u)\end{array}$ & $\begin{array}{l}C_{V} \text { with } \\
k_{o p}(u)\end{array}$ \\
\hline 0 & 1 & 0.8695 & 0.8944 & 6.666 & 637.03 & 3.78 \\
4 & 0.8375 & 0.5094 & 0.5122 & 40.004 & 5245.04 & 1.81 \\
8 & 0.7955 & 0.2926 & 0.2934 & 80.011 & 11581.0 & 1.34 \\
12 & 0.7825 & 0.1677 & 0.1680 & 120.008 & 17968.9 & 1.11 \\
16 & 0.7761 & 0.0961 & 0.0962 & 160.034 & 24380.4 & 0.97 \\
20 & 0.7724 & 0.0550 & 0.0551 & 200.011 & 30783.9 & 0.87 \\
\hline
\end{tabular}

Observe that the probabilities of ruin obtained are lower with $k_{o p}(u)$ than with $k=$ 0.7577. At higher levels of $u$, the probabilities of ruin tend towards the same value; clearly the exact value obtained with the minimization of expression (33) tends towards the upper bound of the probability of ruin.

If we assume that the amount is Erlang $(2,2)$; the optimal policy with proportional reinsurance is shown in Table 7.

Table 7 Minimal probability of ruin with proportional reinsurance with $k_{o p}(u)$ if $X \sim \operatorname{Erlang}(2,2)$

\begin{tabular}{lll}
\hline$u$ & $k_{o p}(u)$ & $\psi_{\min }^{k_{o p}}(u)$ \\
\hline 0 & 1 & 0.869565 \\
4 & 0.81269 & 0.425417 \\
8 & 0.786636 & 0.200804 \\
12 & 0.778327 & 0.0946819 \\
16 & 0.77424 & 0.0446321 \\
20 & 0.771808 & 0.0210369 \\
\hline
\end{tabular}

After considering the optimal strategy with proportional reinsurance, we compare the results obtained with the optimal strategy in threshold reinsurance. To do so, we calculate $d(u)=\psi_{\min }^{k_{o p}}(u)-\psi_{\min }^{k_{1} \neq k_{2}}(u)$ as a function that allows us to compare $\psi_{\min }^{k_{1} \neq k_{2}}(u)$, the results of which were shown in Tables 4 and 5, with the optimal results in a strategy of proportional reinsurance, $\psi_{\min }^{k_{o p}}(u)$, obtained in Tables 6 and 7. Fig. 2 shows $d(u)$ for $X \sim \operatorname{Exp}(1)$ and $X \sim \operatorname{Erlang}(2,2)$, 


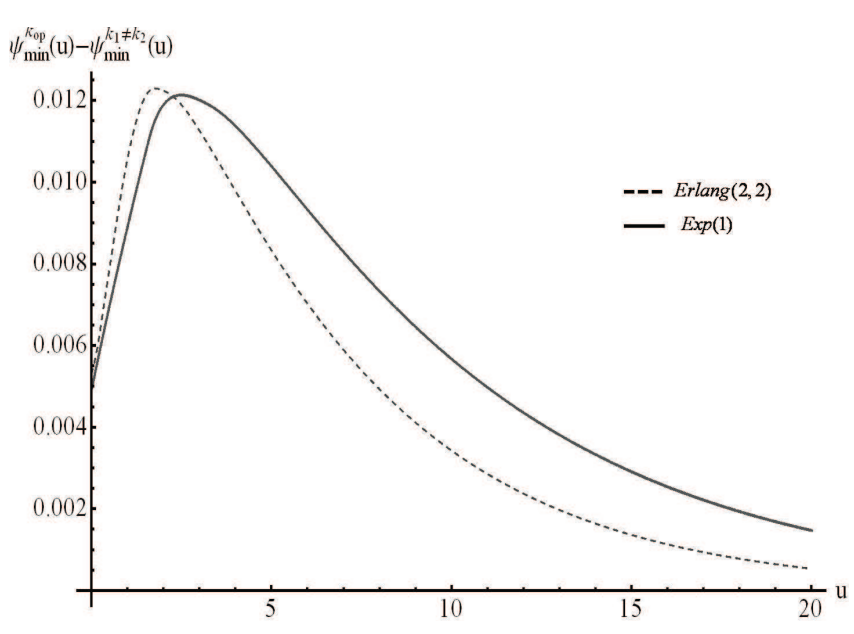

Fig. $2 d(u)=\psi_{\min }^{k_{o p}}(u)-\psi_{\min }^{k_{1} \neq k_{2}}(u)$ for $X \sim \operatorname{Exp}(1)$ and $X \sim \operatorname{Erlang}(2,2)$

Observe that for example for $X \sim \operatorname{Exp}(1)$, the difference between the minimum probabilities of ruin with proportional reinsurance and threshold reinsurance respectively with $b=3.27$ increases for small values of $u$, reaching a maximum and then decreasing as the initial value of the reserves increases. Therefore, the threshold reinsurance strategy always produces better results in terms of probability of ruin than the proportional reinsurance strategy for any initial level of the reserves. The behaviour if $X \sim \operatorname{Erlang}(2,2)$ is similar.

With threshold proportional reinsurance, the insurer can reduce his probability of ruin for some predetermined level of initial capital relative to the options of applying proportional reinsurance. This optimality of threshold proportional reinsurance also implies that, if the manager wants to obtain this minimal probability of ruin but with proportional reinsurance more initial capital will be needed. The relative increase in the initial reserves to achieve this optimal probability of ruin can be considered as the cost of the options of proportional reinsurance against threshold proportional reinsurance.

Assuming $X \sim \operatorname{Exp}(1)$, Table 8 shows, for different values of the initial level of the reserves, the minimal probability of ruin. As we have seen above, it is obtained with the threshold reinsurance strategy. We calculate the initial level of reserves needed to obtain this probability in a model with proportional reinsurance and the relative cost for the insurer of choosing proportional reinsurance rather than threshold reinsurance,

Table 8 For $X \sim \operatorname{Exp}(1)$, relative cost in $u$ if proportional reinsurance is chosen

\begin{tabular}{llll}
\hline$\psi_{\min }^{k_{1} \neq k_{2}}$ & $\begin{array}{l}\text { threshold } \\
\text { reinsurance } \\
u(1)\end{array}$ & $\begin{array}{l}\text { proportional } \\
\text { reinsurance } \\
u(2)\end{array}$ & $\frac{(2)-(1)}{(1)}$ \\
\hline 0.864665 & 0 & 0.043 & \\
0.498067 & 4 & 4.164 & 0.04091 \\
0.285276 & 8 & 8.182 & 0.02282 \\
0.163396 & 12 & 12.189 & 0.01575 \\
0.093587 & 16 & 16.192 & 0.01201 \\
0.053603 & 20 & 20.194 & 0.00971 \\
\hline
\end{tabular}


Observe, for example, that for $u=4$, if the manager chooses threshold proportional reinsurance he achieves a probability of ruin of 0.498 . If he chooses proportional reinsurance, always with 0.8375 as the retention percentage, to achieve this probability of ruin he will need $4.091 \%$ more initial capital.

\section{Appendix}

\section{A Proof of Theorem 1}

Let us decompose every inter-occurrence time with generalized $\operatorname{Erlang}(n)$-distribution into the independent sum of $n$ exponential random variables with parameters $\lambda_{1}, \ldots, \lambda_{n}$, each causing a "sub-claim" of size 0 . At the time of the $n$th sub-claim an actual claim with distribution function $F$ occurs. This can be achieved by considering $n$ states of the risk process. Starting at time 0 in state 1 , every sub-claim causes a transition to the next state and the time of occurrence of the $n$th sub-claim, an actual claim with distribution $F$ occurs and the risk process jumps into state 1 again.

This will allow us to use Markovian arguments due to the lack-of-memory property of the exponential distribution.

Let $\phi_{i}^{j)}(u)$ denote the Gerber-Shiu function (4) if the risk process is in state $j$ ( $j=$ $1, \ldots, n)$.

Indeed, conditioning on the occurrence of a sub-claim, we obtain for $0 \leq u<b$, for $j=1, \ldots, n-1$

$$
\phi_{i}^{j)}(u)=\left(1-\lambda_{j} d t\right) e^{-\delta d t} \phi_{i}^{j)}\left(u+c_{i} d t\right)+\lambda_{j} d t e^{-\delta d t} \phi_{i}^{j+1)}\left(u+c_{i} d t\right)+o(d t)
$$

being $i=1$ for $0 \leq u<b$ and $i=2$ for $u \geq b$.

From (35) we obtain, by Taylor expansion and collecting all terms of order $d t$, for $j=$ $1, \ldots, n-1$,

$$
-\left(\delta+\lambda_{j}\right) \phi_{i}^{j)}(u)+c_{i} \phi_{i}^{j) \prime}(u)+\lambda_{j} \phi_{i}^{j+1)}(u)=0,
$$

and following a similar process for $j=n$,

$$
\begin{aligned}
& \left(c_{1} \frac{d \cdot}{d u}-\left(\lambda_{n}+\delta\right)\right) \phi_{1}^{n)}(u)+\lambda_{n} \int_{0}^{\frac{u}{k_{1}}} \phi_{1}^{1)}\left(u-x k_{1}\right) d F(x) \\
& +\lambda_{n} \int_{\frac{u}{k_{1}}}^{\infty} w\left(u, x k_{1}-u\right) d F(x)=0, \\
& \left(c_{2} \frac{d \cdot}{d u}-\left(\lambda_{n}+\delta\right)\right) \phi_{2}^{n)}(u)+\lambda_{n}\left[\int_{0}^{\frac{u-b}{k_{2}}} \phi_{2}^{1)}\left(u-x k_{2}\right) d F(x)\right. \\
& \left.+\int_{\frac{u-b}{k_{2}}}^{\frac{u}{k_{2}}} \phi_{1}^{1)}\left(u-x k_{2}\right) d F(x)+\int_{\frac{u}{k_{2}}}^{\infty} w\left(u, x k_{2}-u\right) d F(x)\right]=0 .
\end{aligned}
$$

From (36),

$$
\phi_{i}^{j+1)}(u)=\frac{\left(\delta+\lambda_{j}\right)-c_{i} \frac{d \cdot}{d u}}{\lambda_{j}} \phi_{i}^{j)}(u), \quad j=1, \ldots, n-1
$$


SO

$$
\phi_{i}^{n)}(u)=\left(\prod_{j=1}^{n-1} \frac{\delta+\lambda_{j}-c_{i} \frac{d \cdot}{d u}}{\lambda_{j}}\right) \phi_{i}^{1)}(u)
$$

and substituting (40) in (37) and in (38) yields (5) and (6).

By the continuity argument $\left.\phi_{1}^{j)}(u)\right|_{u=b^{-}}=\phi_{2}^{j)}(b)$. Therefore, for $j=1, \ldots, n-1$, from (36)

$$
c_{1} \phi_{1}^{j) \prime}(b)=c_{2} \phi_{2}^{j) \prime}(b)
$$

and for $j=n$, from (37) and (38),

$$
\begin{aligned}
& c_{1} \phi_{1}^{n) \prime}(b)+\lambda_{n} \int_{0}^{\frac{b}{k_{1}}} \phi_{1}^{1)}\left(b-x k_{1}\right) d F(x)+\lambda_{n} \int_{\frac{b}{k_{1}}}^{\infty} w\left(b, x k_{1}-b\right) d F(x) \\
= & c_{2} \phi_{2}^{n) \prime}(b)+\lambda_{n} \int_{0}^{\frac{b}{k_{2}}} \phi_{1}^{1)}\left(b-x k_{2}\right) d F(x)+\lambda_{n} \int_{\frac{b}{k_{2}}}^{\infty} w\left(b, x k_{2}-b\right) d F(x) .
\end{aligned}
$$

From (41) and (42), and using (39) and (40), (7) is obtained.

\section{B Proof of Theorem 2}

From (14), it is easy to obtain

$$
\begin{gathered}
f^{(N)}(x)=-\frac{1}{b_{N}} \sum_{i=0}^{N-1} b_{i} f^{(i)}(x), \\
f^{(N+1)}(x)=-\frac{1}{b_{N}} \sum_{i=0}^{N} b_{i-1} f^{(i)}(x), \\
1-b_{1} f(0)-b_{2} f^{\prime}(0)-\ldots-b_{N} f^{(N-1)}(0)=0, \\
F(x)=1-\sum_{i=1}^{N} b_{i} f^{(i-1)}(x) .
\end{gathered}
$$

For $0<u<b$, we need some previous results,

Definition $1 I N_{h}$ is the $h$-th integral,

$$
I N_{h}=\int_{0}^{\frac{u}{k_{1}}} \phi_{1}\left(u-x k_{1}\right) f^{(h)}(x) d x,
$$

being $h=0, \ldots, N$ and $f^{(0)}(x)=f(x)$.

Lemma 1 The derivative of $I N_{h}$ with respect to $u$ is

$$
I N_{h}^{\prime}=\frac{f^{(h)}(0)}{k_{1}} \phi_{1}(u)+\frac{I N_{h+1}}{k_{1}} .
$$


Proof From Definition 1, using the Leibniz rule

$$
I N_{h}^{\prime}=\frac{\phi_{1}(0) f^{(h)}\left(\frac{u}{k_{1}}\right)}{k_{1}}+\int_{0}^{\frac{u}{k_{1}}} f^{(h)}(x) \frac{\partial \phi_{1}\left(u-x k_{1}\right)}{\partial u} d x,
$$

and solving by parts the integral of (48),

$$
I N_{h}^{\prime}=\frac{\phi_{1}(u) f^{(h)}(0)}{k_{1}}+\frac{1}{k_{1}} \int_{0}^{\frac{u}{k_{1}}} \phi_{1}\left(u-x k_{1}\right) f^{(h+1)}(x) d x .
$$

Taking into account Definition 1 and (49), the proof is completed.

Lemma 2 The h-th derivative of IN $N_{0}$ with respect to $u$ is

$$
I N_{0}^{(h)}=\frac{I N_{h}}{k_{1}^{h}}+\sum_{s=0}^{h-1} \frac{\phi_{1}^{(s)}(u)}{k_{1}^{h-s}} f^{(h-1-s)}(0),
$$

where $1 \leq h \leq N$.

Proof $I N_{0}^{(h)}$ is the $h$-th derivative of $I N_{0}$ with respect to $u$. It can be proved by induction expression (50).

For $h=1$,

$$
I N_{0}^{\prime}=\frac{f(0)}{k_{1}} \phi_{1}(u)+\frac{I N_{1}}{k_{1}}
$$

so, using Lemma 1 , the validity of Lemma 2 for an initial integer is certain.

For $h+1$, assuming validity for $h$,

$$
I N_{0}^{(h+1)}=\frac{I N_{h}^{\prime}}{k_{1}^{h}}+\sum_{s=0}^{h-1} \frac{\phi_{1}^{(s+1)}(u)}{k_{1}^{h-s}} f^{(h-1-s)}(0),
$$

and using Lemma 1 we obtain,

$$
\begin{aligned}
I N_{0}^{(h+1)} & =\frac{1}{k_{1}^{h}}\left(\frac{f^{(h)}(0)}{k_{1}} \phi_{1}(u)+\frac{I N_{h+1}}{k_{1}}\right)+\sum_{s=0}^{h-1} \frac{\phi_{1}^{(s+1)}(u)}{k_{1}^{h-s}} f^{(h-1-s)}(0) \\
& =\frac{f^{(h)}(0)}{k_{1}^{h+1}} \phi_{1}(u)+\frac{I N_{h+1}}{k_{1}^{h+1}}+\sum_{s=0}^{h-1} \frac{\phi_{1}^{(s+1)}(u)}{k_{1}^{h-s}} f^{(h-1-s)}(0) \\
& =\frac{f^{(h)}(0)}{k_{1}^{h+1}} \phi_{1}(u)+\frac{I N_{h+1}}{k_{1}^{h+1}}+\sum_{s=1}^{h} \frac{\phi_{1}^{(s)}(u)}{k_{1}^{h-s+1}} f^{(h-s)}(0),
\end{aligned}
$$

and simplifying

$$
I N_{0}^{(h+1)}=\frac{I N_{h+1}}{k_{1}^{h+1}}+\sum_{s=0}^{h} \frac{\phi_{1}^{(s)}(u)}{k_{1}^{h+1-s}} f^{(h-s)}(0),
$$

then, expression (50) is valid for $h+1$. So, by mathematical induction, $I N_{0}^{(h)}$ from Lemma 2 is certain for all integer $h, 1 \leq h \leq N$.

Lemma $3 I N_{N}$ can be written as

$$
I N_{N}=-\frac{1}{b_{N}} \sum_{h=0}^{N-1} b_{h} I N_{h}
$$


Proof From Definition 1

$$
I N_{N}=\int_{0}^{\frac{u}{k_{1}}} \phi_{1}\left(u-x k_{1}\right) f^{(N)}(x) d x
$$

substituting (43) in (52)

$$
\begin{aligned}
I N_{N} & =-\frac{1}{b_{N}} \int_{0}^{\frac{u}{k_{1}}} \phi_{1}\left(u-x k_{1}\right) \sum_{i=0}^{N-1} b_{i} f^{(i)}(x) d x \\
& =-\frac{1}{b_{N}} \sum_{i=0}^{N-1} b_{i} \int_{0}^{\frac{u}{k_{1}}} \phi_{1}\left(u-x k_{1}\right) f^{(i)}(x) d x .
\end{aligned}
$$

The integro-differential equation (11) and its derivatives with respect to $u$ until $N+1$ depending on the derivatives of $I N_{0}$ can be written as

$$
\begin{aligned}
\phi_{1}^{\prime}(u) & =\frac{\lambda+\delta}{c_{1}} \phi_{1}(u)-\frac{\lambda}{c_{1}} I N_{0}-\frac{\lambda}{c_{1}}\left[1-F\left(\frac{u}{k_{1}}\right)\right], \\
\phi_{1}^{(h+1)}(u) & =\frac{\lambda+\delta}{c_{1}} \phi_{1}^{(h)}(u)+\frac{\lambda}{c_{1} k_{1}^{h}} f^{(h-1)}\left(\frac{u}{k_{1}}\right)-\frac{\lambda}{c_{1}} I N_{0}^{(h)}, 1 \leq h \leq N .
\end{aligned}
$$

By Lemma 2,

$$
\begin{aligned}
\phi_{1}^{\prime}(u)= & \frac{\lambda+\delta}{c_{1}} \phi_{1}(u)-\frac{\lambda}{c_{1}} I N_{0}-\frac{\lambda}{c_{1}}\left[1-F\left(\frac{u}{k_{1}}\right)\right], \\
\phi_{1}^{(h+1)}(u)= & \frac{\lambda+\delta}{c_{1}} \phi_{1}^{(h)}(u)+\frac{\lambda}{c_{1} k_{1}^{h}} f^{(h-1)}\left(\frac{u}{k_{1}}\right) \\
& -\frac{\lambda}{c_{1}}\left(\frac{I N_{h}}{k_{1}^{h}}+\sum_{s=0}^{h-1} \frac{\phi_{1}^{(s)}(u)}{k_{1}^{h-s}} f^{(h-1-s)}(0)\right), 1 \leq h \leq N .
\end{aligned}
$$

From (54) and (55),

$$
\begin{aligned}
I N_{0} & =\frac{c_{1}}{\lambda}\left(\frac{\lambda+\delta}{c_{1}} \phi_{1}(u)-\frac{\lambda}{c_{1}}\left[1-F\left(\frac{u}{k_{1}}\right)\right]-\phi_{1}^{\prime}(u)\right) \\
& =\frac{\lambda+\delta}{\lambda} \phi_{1}(u)-\left[1-F\left(\frac{u}{k_{1}}\right)\right]-\frac{c_{1}}{\lambda} \phi_{1}^{\prime}(u), \\
I N_{h}= & \frac{c_{1} k_{1}^{h}}{\lambda}\left(\frac{\lambda+\delta}{c_{1}} \phi_{1}^{(h)}(u)+\frac{\lambda}{c_{1} k_{1}^{h}} f^{(h-1)}\left(\frac{u}{k_{1}}\right)\right. \\
& \left.-\frac{\lambda}{c_{1}} \sum_{s=0}^{h-1} \frac{\phi_{1}^{(s)}(u)}{k_{1}^{h-s}} f^{(h-1-s)}(0)-\phi_{1}^{(h+1)}(u)\right), 1 \leq h \leq N .
\end{aligned}
$$


Substituting (56) and (57) in (51),

$$
\begin{aligned}
I N_{N}= & -\frac{1}{b_{N}} I N_{0}-\frac{1}{b_{N}} \sum_{h=1}^{N-1} b_{h} I N_{h} \\
= & -\frac{(\lambda+\delta)}{b_{N} \lambda} \phi_{1}(u)+\frac{1}{b_{N}}\left[1-F\left(\frac{u}{k_{1}}\right)\right]+\frac{c_{1}}{b_{N} \lambda} \phi_{1}^{\prime}(u) \\
& -\frac{1}{b_{N}} \sum_{h=1}^{N-1} \frac{b_{h} k_{1}^{h}(\lambda+\delta)}{\lambda} \phi_{1}^{(h)}(u)-\frac{1}{b_{N}} \sum_{h=1}^{N-1} b_{h} f^{(h-1)}\left(\frac{u}{k_{1}}\right) \\
& +\frac{1}{b_{N}} \sum_{h=1}^{N-1} b_{h} \sum_{s=0}^{h-1} \phi_{1}^{(s)}(u) k_{1}^{s} f^{(h-1-s)}(0)+\frac{1}{b_{N}} \sum_{h=1}^{N-1} \frac{b_{h} c_{1} k_{1}^{h}}{\lambda} \phi_{1}^{(h+1)}(u) .
\end{aligned}
$$

Substituting (46) in (58) and rearranging terms,

$$
I N_{N}=f^{(N-1)}\left(\frac{u}{k_{1}}\right)+\sum_{s=0}^{N} \phi_{1}^{(s)}(u) D_{s}
$$

with

$$
D_{s}= \begin{cases}\frac{1}{b_{N}} \sum_{h=1}^{N-1} b_{h} f^{(h-1)}(0)-\frac{\lambda+\delta}{b_{N} \lambda}, & s=0 \\ \frac{c_{1} b_{s-1} k_{1}^{s-1}}{b_{N} \lambda}-\frac{(\lambda+\delta) b_{s} k_{1}^{s}}{b_{N} \lambda}+\frac{k_{1}^{s}}{b_{N}} \sum_{h=s+1}^{N-1} b_{h} f^{(h-1-s)}(0), & s=1, \ldots, N-1 \\ \frac{c_{1} b_{N-1} k_{1}^{N-1}}{b_{N} \lambda}, & s=N .\end{cases}
$$

Finally, substituting (59) in (55) and taking into account relation (45),

$$
\begin{aligned}
\phi_{1}^{(N+1)}(u)= & \frac{\lambda+\delta}{c_{1}} \phi_{1}^{(N)}(u)-\frac{\lambda}{c_{1} k_{1}^{N}} \sum_{s=0}^{N} \phi_{1}^{(s)}(u) D_{s} \\
& -\frac{\lambda}{c_{1}} \sum_{s=0}^{N-1} \frac{\phi_{1}^{(s)}(u)}{k_{1}^{N-s}} f^{(N-1-s)}(0) \\
= & \left(\frac{\lambda+\delta}{c_{1}}-\frac{b_{N-1}}{k_{1} b_{N}}\right) \phi_{1}^{(N)}(u)+\frac{\delta}{c_{1} b_{N} k_{1}^{N}} \phi_{1}(u) \\
& -\sum_{s=1}^{N-1}\left(\frac{b_{s-1}}{k_{1} b_{N}}-\frac{(\lambda+\delta) b_{s}}{c_{1} b_{N}}+\frac{\lambda}{c_{1} b_{N}} \sum_{h=s+1}^{N-1} b_{h} f^{(h-1-s)}(0)\right. \\
& \left.+\frac{\lambda}{c_{1}} f^{(N-1-s)}(0)\right) \frac{1}{k_{1}^{N-s}} \phi_{1}^{(s)}(u) .
\end{aligned}
$$

For $u>b$, we can obtain $\phi_{2}^{(N+1)}(u)$ by an analogous process substituting $c_{1}, k_{1}$ and $\phi_{1}(u)$ by $c_{2}, k_{2}$ and $\phi_{2}(u)$. 


\section{References}

Albrecher H, Claramunt MM, Mármol M (2005) On the distribution of dividend payments in a Sparre Andersen model with generalized Erlang(n) interclaim times. Insur Math Econ 37(2):324-334

Bowers NL, Gerber HU, Hickman JC, Jones DA, Nesbitt CJ (1997) Actuarial mathematics. Society of Actuaries, Illinois

Bühlmann H (1996) Mathematical methods in risk theory. Springer-Verlag, Berlin

Centeno L (1986) Measuring the effects of reinsurance by the adjustment coefficient. Insur Math Econ 5(2):169-182

Centeno L (2002) Measuring the effects of reinsurance by the adjustment coefficient in the Sparre Anderson model. Insur Math Econ 30(1):37-49

Centeno L (2005) Dependent risks and excess of loss reinsurance. Insur Math Econ 37(2):229-238

Dickson DCM (2005) Insurance risk and ruin. Cambridge University press, United Kingdom

Dickson DCM, Drekic S (2004) The joint distribution of the surplus prior to ruin and the deficit at ruin in some Sparre Andersen models. Insur Math Econ 34(1):97-107

Dickson DCM, Waters HR (1996) Reinsurance and ruin. Insur Math Econ 19(1):61-80

Dickson DCM, Waters HR (2006) Optimal dynamic reinsurance. Astin Bull 36(2):415-432

Gerber HU (1979) An introduction to mathematical risk theory. S.S. Huebner Foundation monograph, University of Pennsylvania, Philadelphia

Gerber HU, Shiu ES (1998) On the time value of ruin. N Am Actuar J 2(1):48-78

Gerber HU, Shiu ES (2005) The time value of ruin in a Sparre Andersen model. N Am Actuar J 9(2):49-84

Goovaerts MJ, Van Heervaarden AE, Kaas R (1989) Optimal reinsurance in relation to ordering of risk. Insur Math Econ 8(1):11-17

Hesselager O (1990) Some results on optimal reinsurance in terms of the adjustment coefficient. Scand Actuar J 1990(1-2):80-95

Hipp C (2006) Speedy convolution algorithms and Panjer recursions for phase-type distributions. Insur Math Econ 38(1): 176-188

Hipp C, Vogt M (2003) Optimal dynamic XL reinsurance. Astin Bull 33(2):193-207

Hojgaard B, Taksar M (1998) Optimal proportional reinsurance policies for diffusion models with transaction costs. Insur Math Econ 22(1):41-51

Landriault D, Willmot GE (2008) On the Gerber-Shiu discounted penalty function in the Sparre Andersen model with an arbitrary interclaim time distribution. Insur Math Econ 42(2):600-608

Li S, Garrido J (2004) On ruin for the Erlang(n) risk process. Insur Math Econ 34(3):391-408

Lin XS, Pavlova K (2006) The compound Poisson risk model with a threshold dividend strategy. Insur Math Econ 38(1):57-80

Lin XS, Willmot GE (1999) Analysis of a defective renewal equation arising in ruin theory. Insur Math Econ 25(1):63-84

Lin XS, Willmot GE (2000) The moments of the time of ruin, the surplus before ruin, and the deficit at ruin. Insur Math Econ 27(1):19-44

Schmidli H (2001) Optimal proportional reinsurance policies in a dynamic setting. Scand Actuar J 2001(1):55-68

Schmidli H (2002) On minimizing the ruin probability by investment and reinsurance. Ann Appl Probab 12(3):890-907

Schmidli H (2006) Optimisation in non-life insurance. Stoch Models 22(4):689-722

Taksar M, Markussen C (2003) Optimal dynamic reinsurance policies for large insurance portfolios. Finance Stoch 7(1):97-121

Verlaak R, Beirlant J (2003) Optimal reinsurance programs: an optimal combination of several reinsurance protections on a heterogeneous insurance portfolio. Insur Math Econ 33(2):381-403

Waters HR (1979) Excess of loss reinsurance limits. Scand Actuar J 1979(1):37-43

Waters HR (1983) Some mathematical aspects of reinsurance. Insur Math Econ 2(1):17-26

Willmot GE (2007) On the discounted penalty function in the renewal risk model with general interclaim times. Insur Math Econ 41(1):17-31 